\title{
SÍNDROME DE STEVENS JOHNSON ASSOCIADA AO USO DE FENITOÍNA: UM RELATO DE CASO
}

CORREGLIANO, Mariana Rossi ${ }^{1}$ MARTINS, Stella de Souza ${ }^{2}$ PINTO, Luelen da Silva ${ }^{3}$

RESUMO: A síndrome de Stevens-Johnson é uma afecção dermatológica rara e grave, que se caracteriza por um processo de necrose dos queratinócitos e consequente descolamento da epiderme. A maioria dos casos ocorre devido ao uso de fármacos, principalmente beta-lactâmicos, anticonvulsivantes aromáticos e alopurinol. O diagnóstico é basicamente clínico e o tratamento é focado na retirada do agente causador associado a suporte clínico. Este trabalho tem como objetivo principal contribuir para a difusão de conhecimento médico a respeito da síndrome em questão, através da descrição de um caso clínico de paciente internada em uma Santa Casa no interior do estado de São Paulo no ano de 2019.

Palavras-chave: Anticonvulsivante. Necrose. Epiderme.

\section{STEVENS JOHNSON SYNDROME ASSOCIATED WITH THE USE OF PHENYTOIN: A CASE REPORT}

SUMMARY: Stevens-Johnson's syndrome is a rare dermatological entity and potentially fatal, which is characterized by a process of necrosis of the keratinocytes and detachment of the skin. The majority of the cases occur due to the use of pharmacological drugs, mainly beta-lactams, aromatic anticonvulsants and allopurinol. The diagnosis is basically clinical and the treatment is focused not only on removing the drug responsible for the disease but also with clinical support. This report has as its main goal to contribute to medical awareness about the syndrome mentioned through the description of a clinical case of a patient admitted in Santa Casa de Franca in 2019.

Keywords: Anticonvulsant. Necrosis. Skin.

\section{INTRODUÇÃO}

Descrita pela primeira vez no ano de 1922, a Síndrome de Stevens-Johnson é uma entidade dermatológica rara e potencialmente fatal, caracterizada por um processo de necrose dos queratinócitos e consequente descolamento da epiderme. (OLIVEIRA; SANCHES; SELORES, 2011; RIBEIRO; RIBEIRO; BENITO, 2017). Apresenta incidência anual média de 1,2 - 6 casos/milhão de habitantes, com destaque em adultos do sexo masculino, imunossuprimidos e

\footnotetext{
${ }^{1}$ Graduando em Medicina. Faculdade de Medicina de Franca- UNIFRAN - ORCIDhttps://orcid.org/0000-0002-3475-9248

${ }^{2}$ Graduando em Medicina. Faculdade de Medicina de Franca- UNIFRAN - ORCIDhttps://orcid.org/0000-0002-3835-6913

${ }^{3}$ Médica-residente de clínica médica da Santa Casa de Misericórdia de Franca SP
} 
portadores de tumor cerebral em tratamento concomitante com radioterapia e anticonvulsivantes (ROCHA, et al., 2018; BULISANI, et al., 2006).

Cerca de $80 \%$ dos casos estão associados ao uso de alguns grupos de fármacos, sendo os beta-lactamicos, sulfonamidas, anticonvulsivantes aromáticos (como a fenitoína e o fenobarbital), AINEs e o Alopurinol os principais. Também apresenta relação com infecções por agentes como herpes vírus e Mycoplasma (OLIVEIRA; SANCHES; SELORES, 2011; BULISANI, et al., 2006). Acredita-se que seu desenvolvimento ocorra pela combinação da exposição a esses fatores com condições genéticas preexistentes (como defeitos no gene HLA e deficiências enzimáticas) (BULISANI, et al., 2006).

O surgimento dos sintomas acontece, em média, 21 dias após contato com o agente desencadeante. Apresenta pródromo marcado por sintomas gerais (febre, mal-estar, mialgia e artralgia) seguido de erupções cutâneas não pruriginosa, confluentes, que surgem no tronco e logo se espalham, poupando apenas palmas das mãos e plantas dos pés. As lesões evoluem para bolhas, muitas delas com centro necrótico, e descolamento da epiderme, que pode vir associado a sangramentos. Formam-se lesões erosivas de extensão variada e risco elevado de infecção (OLIVEIRA; SANCHES; SELORES, 2011; OLIVEIRA et al., 2012).

Regiões de mucosa também podem ser acometidas, como mucosa oral, ocular, genital e de trato respiratório, causando sintomas como disfagia, fotofobia, disúria e, nos casos mais graves, até mesmo edema de interstício pulmonar com hipoxemia e dispneia. (OLIVEIRA; SANCHES; SELORES, 2011) A evolução para cenários agressivos ocorre principalmente nos grupos de risco, sendo eles: maiores de 40 anos, portadores de neoplasia, descolamento de mais de $10 \%$ da epiderme, taquicardia como sinal clínico e alterações laboratoriais específicas (ureia acima de $28 \mathrm{mg} / \mathrm{dL}$; glicose acima de $252 \mathrm{mg} / \mathrm{dL}$, bicarbonato sérico abaixo de $20 \mathrm{mg} / \mathrm{dL}$ ) (OLIVEIRA; SANCHES; SELORES, 2011; SANT; DIAS; AVANCINI, 2016).

O diagnóstico é essencialmente clínico, mas pode ser confirmado com biópsia cutânea (SANT; DIAS; AVANCINI, 2016). É importante que seja feito precocemente para que medidas terapêuticas sejam tomadas antes do desenvolvimento de complicações agudas - como distúrbios hidroeletrolíticos, hipotermia e infecções - e crônicas, como sinéquias e estenoses (OLIVEIRA; SANCHES; SELORES, 2011).

O item mais importante do conjunto de medidas terapêuticas para a síndrome é a retirada do fármaco causador. Medidas de suporte, como nutrição adequada, manutenção da temperatura, reposição de fluidos e eletrólitos e analgesia devem ser adotadas conforme a necessidade. Não há indicação de antibioticoprofilaxia. Corticoides, imunossupressores e plasmaférese são opções terapêuticas em estudo. Atualmente, o uso de imunoglobulina é o que mais se associa a redução 
das taxas de mortalidade (OLIVEIRA; SANCHES; SELORES, 2011; ARRUDA; SAMPAIO, 2014).

A síndrome de Stevens Johnson é uma patologia rara, porem seu diagnóstico precoce é imperativo, pois está associada a elevada morbimortalidade. Portanto, é imprescindível um melhor conhecimento da doença para instituir o tratamento adequado. Desta forma, este estudo tem o intuito de contribuir com informações, de forma a permitir um melhor conhecimento quanto ao diagnóstico e manejo clínico desta patologia.

\section{DESCRIÇÃO DO CASO}

Paciente do sexo feminino, 27 anos, encaminhada para internação hospitalar devido a um quadro de exantema generalizado, não pruriginoso, que iniciou em tórax, progrediu para abdome, membros e face, e evoluiu com a formação de bolhas. Também referia ardência ocular e hiperemia conjuntival associadas a secreção amarela. Apresentou um pico febril no dia anterior a internação.

Paciente estava em tratamento de tumor de tronco cerebral há 01 mês, com quimioterapia (Temodal®) e radioterapia. Também estava em uso de fenitoína 100mg 2 vezes ao dia e dexametasona 4mg 3 vezes ao dia, para profilaxia de crise convulsiva, ambos iniciados há 1 mês. Ao exame físico apresentava exantema maculopapular em tórax, abdome, braços, coxas e face; pústulas em região da face e tórax anterior e bolhas com conteúdo amarelo citrino em membros superiores.

Figura 1: Membro superior esquerdo com eritema e bolhas

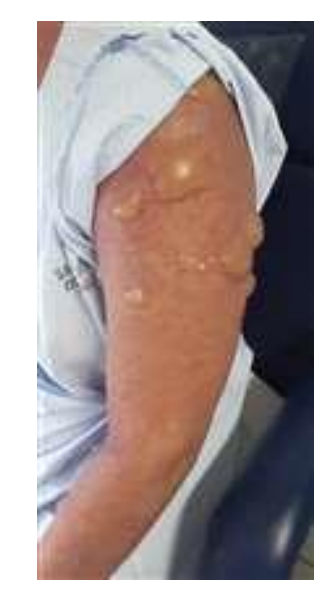

Fonte: Arquivo Pessoal 
Figura 2: Face com lesões eritematosas e bolhosas

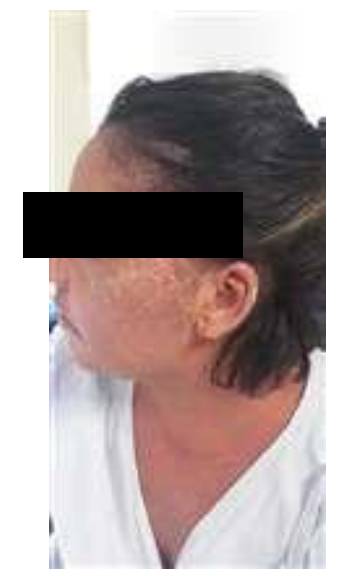

Fonte: Arquivo Pessoal

Foi suspenso o uso de fenitoína e dexametasona e iniciado oxacilina para profilaxia de infecção secundária por 10 dias. Apesar da conduta tomada, a paciente apresentou progressão do exantema para formação de bolhas que evoluíam para rompimento e desprendimento da epiderme, o que causou dor intensa, com necessidade de uso de morfina.

No $3^{\circ}$ dia de internação surgiram lesões ulceradas em regiões oral e genital, levando a piora das queixas álgicas da paciente, com necessidade de introdução de sondas nasogástrica e vesical de demora. Paciente apresentou febre até o $12^{\circ}$ dia de internação.

Figura 3: Membros inferiores com eritema e bolhas extensas

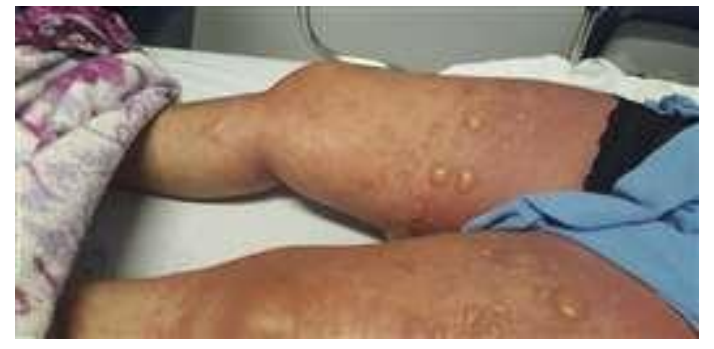

Fonte: Arquivo Pessoal

Figura 4: Membro inferior esquerdo com bolhas com conteúdo amarelo citrino

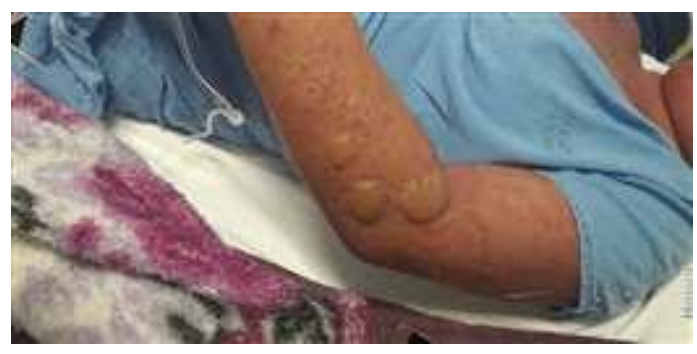

Fonte: Arquivo Pessoal 
Figura 5: Lesões em lábios e mucosa oral

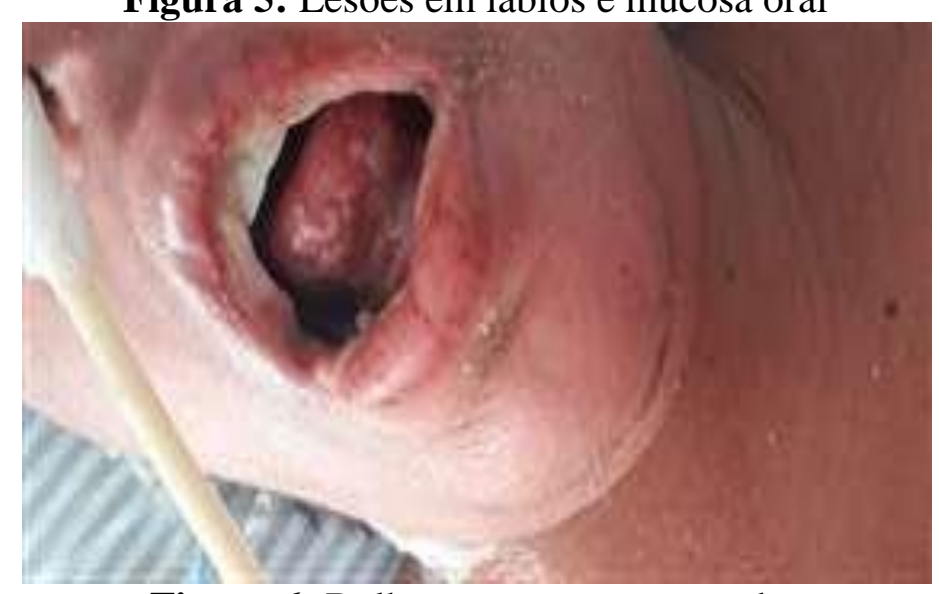

Figura 6: Bolhas extensas e agrupadas

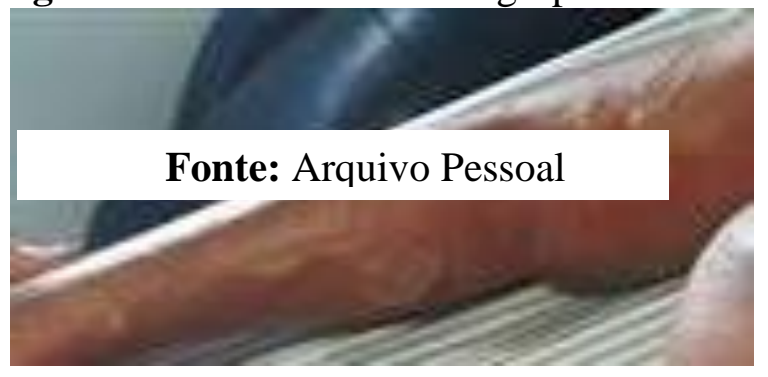

Fonte: Arquivo Pessoal

Com o passar dos dias e manutenção das condutas, houve melhora das lesões, com cicatrização e descamação da pele, e regressão das lesões orais e genitais. A paciente recebeu alta no $16^{\circ}$ dia de internação, assintomática.

Figura 7: início da melhora das lesões após instituição do tratamento

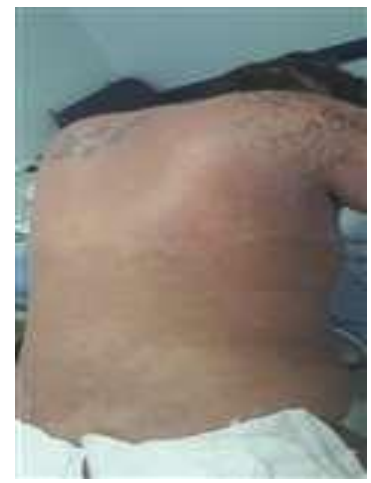

Fonte: Arquivo Pessoal 
Figura 8: Evolução das lesões bolhosas de membro inferior após tratamento

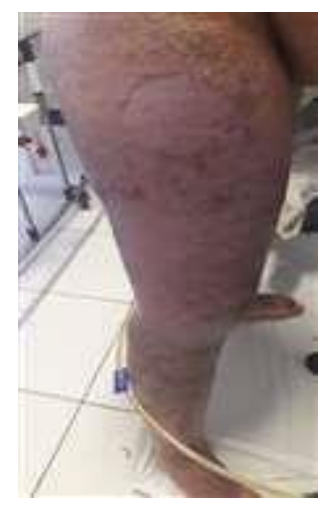

Fonte: Arquivo Pessoal

\section{DISCUSSÃO}

No caso relatado, apesar da paciente não se enquadrar no gênero de maior incidência, diversos aspectos presentes são descritos na literatura como possíveis causas para o desenvolvimento da síndrome: encontra-se na faixa etária de maior risco (segunda e terceira décadas de vida), apresenta imunossupressão e neoplasia de sistema nervoso central tratada com radioterapia associada a anticonvulsivante, sendo o último, ainda, pertencente à classe dos anticonvulsivantes aromáticos, considerada como importante desencadeante do quadro. Nota-se, também, compatibilidade do tempo de exposição à droga com o intervalo médio mencionado na literatura como necessário para considerar uma relação causal (BULISANI, et al., 2006; BASTUJI-GARINS, et al., 1993).

O mecanismo fisiopatológico responsável pelo desenvolvimento das manifestações é incerto. Acredita-se que seja uma reação de hipersensibilidade tardia em resposta a fármacos, infecções ou neoplasias, mediada por linfócitos $\mathrm{T}$ citotóxicos e fator de necrose tumoral-alfa que levam à deposição de imunoglobulinas na junção dermo-epidérmica e induzem a apoptose maciça e posterior necrose das células epiteliais (BULISANI, et al., 2006; MARTÍNEZ-CABRIALES et al., 2015).

Assim como em 90\% dos casos, a paciente acima apresentou acometimento mucoso extenso e doloroso, porém não hemorrágico. Seu diagnóstico foi feito clinicamente, baseado nas características compatíveis com a síndrome, como a febre, as lesões cutâneo - mucosas eritematosas que começam localizadas e evoluem de forma difusa em face, pescoço, dorso e membros superiores, com preservação da palma das mãos e planta dos pés (OLIVEIRA; SANCHES; SELORES, 2011; CHUNG; HUNG, 2010).

Ao mesmo tempo em que a literatura desaconselha o uso de antibioticoprofilaxia nos casos de síndrome de Stevens-Johnson, a presença de febre e leucocitose na grande maioria dos casos pode causar dúvida quanto a presença ou não de uma infecção. Dadas as condições de 
imunossupressão, a permanência prolongada em ambiente hospitalar e a manutenção da febre da paciente do quadro, optou-se pela introdução de oxacilina via oral por 10 dias, sem qualquer tipo aparente de malefício para a evolução do quadro (OLIVEIRA; SANCHES; SELORES, 2011; ARRUDA; SAMPAIO, 2014).

O período de permanência das lesões foi inferior às 6 semanas estimadas na literatura. Apesar da paciente ainda ter apresentado evolução do padrão das lesões após retirada do possível agente causador, o suporte nutricional, hidroeletrolítico e analgésico oferecidos levaram a um desfecho favorável do quadro, sem desenvolvimento de nenhum tipo complicação aguda ou sequela (SOUZA, et al., 2018).

\section{CONCLUSÃO}

Apesar de rara, Stevens-Johnson é uma emergência dermatológica e é muito importante que seu conhecimento seja difundido, pois a realização de um diagnóstico clínico correto e precoce, a aplicação de serviços multidisciplinares e, principalmente, a suspensão precoce do fator causal, são necessários para evitar internações em ambientes de terapia intensiva, para reduzir a necessidade de tratamentos invasivos e para que a diminuição da morbimortalidade seja cada vez mais possível. (PENA. et al., 2020)

Cumpre salientar que a conscientização do paciente a respeito de sua patologia é de fundamental importância para o transcorrer da doença da forma mais adequada e humanitária possível.

Apesar da base do tratamento ser a retirada do agente causador, uma terapia de suporte apropriada e individualizada pode acelerar a recuperação, reduzir o risco de sequelas e melhorar significativamente o prognóstico dos pacientes.

\section{REFERÊNCIAS}

ARRUDA, J. A. A., SAMPAIO, G. C. Síndrome de Stevens-Johnson associada à alopurinol e nimesulida: relato de caso. Revista de Cirurgia de Traumatologia Buco-maxilo. v.14, n.3, Camaragibe Jul./Set. 2014.

BASTUJI-GARIN S., RZANY, B., STERN, R. S., SHEAR, N. H., NALDI, L., ROUJEAU, J. C. Clinical classification of cases of toxic epidermal necrolysis, Stevens-Johnson syndrome, and erythema multiforme. National Library of Medicine. [Online].; Jan 1993, v. 129, n.1, p. 92-6.

BULISANI, A. C. P., SANCHES, G. D., GUIMARÃES, H. P., LOPES, R. D., VENDRAME, L. S, LOPES, A. C. Síndrome de Stevens-Johnson e Necrólise Epidérmica Tóxica em Medicina Intensiva. Revista Brasileira de Terapia Intensiva. v. 18, n. 3, Jul. - Set. 2006. 
CHUNG, W. H., HUNG, S. Genetic markers and danger signals in stevens-johnson syndrome and toxic epidermal necrolysis. National Library of Medicine. [Online].; dez 2010, v. 59, n. 4, $325-32$.

MARTÍNEZ-CABRIALES, A. S., GOMEZ-FLORES, M., OCAMPO-CANDIANI, J. News in severe clinical adverse drug reactions: Stevens-Johnson. Gazeta médica de México. Nov-Dec 2015 , v. 151, n. 6, p.777-87.

OLIVEIRA, A. SANCHES, M. SELORES, M. O espectro clínico síndrome de Stevens-Johnson e necrólise epidérmica tóxica. Acta Médica Portuguesa. 2011. v. 24, n.4, p. 995-1002.

OLIVEIRA, F. L., SILVEIRA, L. K. MORAIS, T. S., SERRA, M. C. V. F. Necrólise epidérmica tóxica e síndrome de Stevens Johnson: atualização. Revista Brasileira de Queimaduras. v.11, n.1, p. 26-30, 2012.

PENA. V. I. et. al. Síndrome de Stevens Johnson após uso de medicação anticonvulsivante - Um relato de caso. Brazilian Journal of Development. Coritiba. v.6. n.10, p.76771-76778. out. 2020.

RIBEIRO. A. G. A., RIBEIRO, M. C., BENITO, L. A. O. Síndrome de Stevens-Johnson(SSJ) em adultos: revisão sistemática. Universitas: Ciências da Saúde. jul/dez; v. 15, n. 2, p. 117-125, 2017.

ROCHA, L. P. S. et al. Relato de caso e Revisão da Literatura de Síndrome de StevensJohnson associada a episódio de varicela. Santa Catarina, julho de 2018. Disponível em < http://www.acm.org.br/acm/seer/index.php/arquivos/article/view/464 > Acesso em: 27 de jan. de 2021.

SANT, C. G., DIAS, N. F., AVANCINI, J. Reações Adversas Medicamentosas. MARTINS, M. A, CARRILHO, F. J., ALVES, V. A. F, CASTILHO, E. A., CERRI, G. G. , editor. Clínica Médica- Alergia e Imunologia. 2nd ed.: Manole; 2016. p. 359-364.

SOUZA, M. C. A., CORREIA, A. P. S., MAGRANI, G. C., JUNIOR, J. C. S. C., CORTES, P. P. R. Síndrome de stevens-johnson e necrólise epidérmica tóxica: relato de caso. Acta biomédica brasiliense. v. 9, n. 1, abr 2018. 